Supporting Information

\title{
Rapid characterization and parameter space exploration of perovskites using an automated routine
}

Elisabeth Reinhardt,,$\uparrow$ Ahmed M. Salaheldin, ${ }^{\dagger}$ Monica Distaso, ${ }^{\dagger}$ Doris Segets, ${ }^{*, \ddagger}$ and Wolfgang Peukert* ${ }^{* \dagger}$

$\dagger$ Institute of Particle Technology (LFG), Interdisciplinary Center for Functional Particle Systems (FPS), Friedrich-Alexander-Universität Erlangen-Nürnberg (FAU), 91058 Erlangen, Germany

$\ddagger$ Process Technology for Electrochemical Functional Materials, Institute for Combustion and Gas Dynamics - Reactive Fluids (IVG-RF), and Center for Nanointegration Duisburg-Essen (CENIDE), University of Duisburg-Essen (UDE), 47057 Duisburg, Germany IAuthors contributed equally

E-mail: doris.segets@uni-due.de; wolfgang.peukert@fau.de

\section{S1 Time effects on precursor formation}

To rule out any time dependencies that could occur during mixing of the two stock solutions and dimethylformamide (DMF), the absorbance peak at $365 \mathrm{~nm}$ which arises due to iodoplumbate $\left(\mathrm{PbI}_{4}^{2-}\right)$ complex formation between MAI and $\mathrm{PbI}_{2}$ was used. ${ }^{1}$ The concen- 
trated MAI and $\mathrm{PbI}_{2}$ solutions in a molar ratio of 1:1 were mixed with DMF by a magnetic stirrer bar at $50{ }^{\circ} \mathrm{C}$. In comparison to the concentration adjusted for the layer production by the automated approach (see manuscript text), the concentration was diluted eightfold to avoid any overloading of the absorption detector. After specific times samples were taken, filled in a $0.5 \mathrm{~mm}$ quartz glass cuvette, and analyzed by UV-Vis absorbance spectroscopy (Perkin Elmer Lambda 35). The therefrom derived absorption spectra for different times are plotted in Figure S1. Obviously, already from the first measuring point at 1 min, homogeneous conditions are achieved and no overlapping time-dependent reactions do occur.

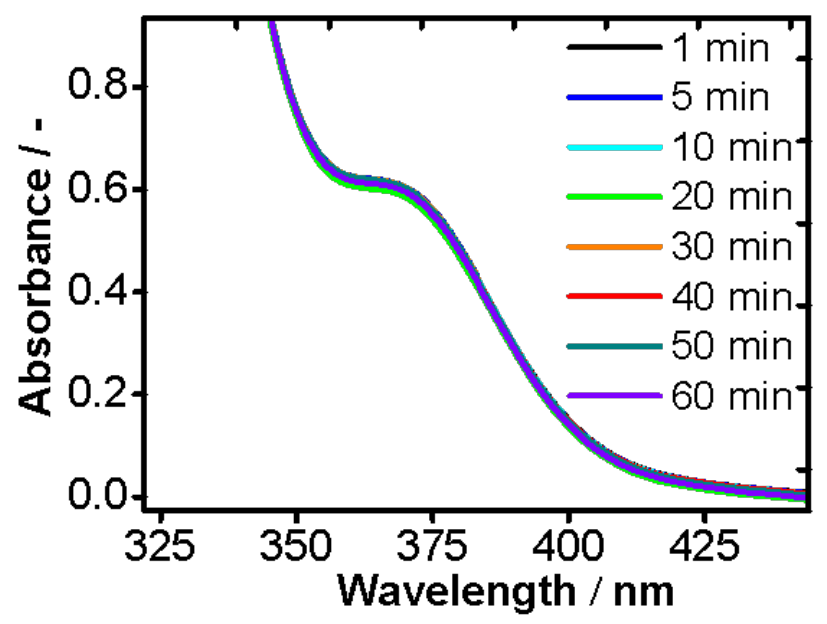

Figure S1: Absorbance spectra for the reactant ratio of 1:1 collected at different times after mixing. 


\section{S2 Determination of annealing time}

By determination of the minimum time needed for annealing, it was possible to reduce significantly the time for perovskite crystallization. The procedure was as follows: first, the prepared glass slides were placed under the objective of a light microscope on a customized $4 \times 5 \mathrm{~cm}^{2}$ heating plate with different temperature set points $\left(40,60,80,100\right.$ and $120{ }^{\circ} \mathrm{C}$, respectively). The mixed precursor solution was deposited in equal volumes, analogous to the automated procedure (see manuscript text). The crystallization process was followed by taking one image per second. This was possible because the $\mathrm{CH}_{3} \mathrm{NH}_{3} \mathrm{PbI}_{3}$ precursor solution had a yellow appearance and could be clearly distinguished from the dark perovskite crystals. The area fraction of the formed perovskite was determined as a function of time and temperature by the software AxioVision (Carl Zeiss Microscopy GmbH). Results of the times which were necessary for complete transformation considering different ratios of $\mathrm{PbI}_{2}: \mathrm{MAI}$ and varying temperatures are summarized in Figure S2.

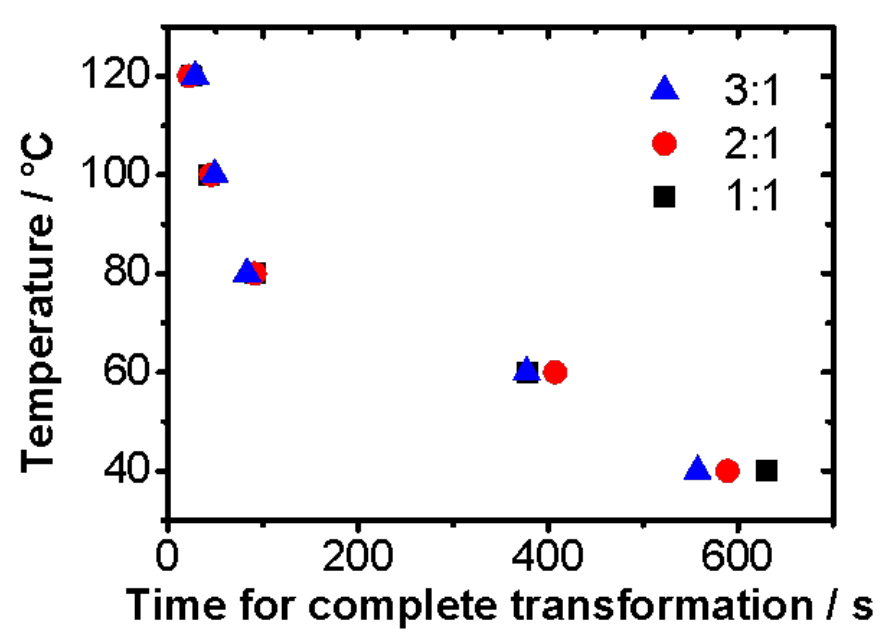

Figure S2: Minimum time that is required to transfer the liquid yellow precursor to a solid, completely dark material. 


\section{S3 Absorption data}

In Figure S3 a)-e) the absorbance spectra for the different ratios and annealing temperatures are summarized. For the sake of clarity, for every synthesis condition one exemplary spectrum is plotted. As reference, also the absorbance spectra of the pure educts are shown in Figure S3f. 

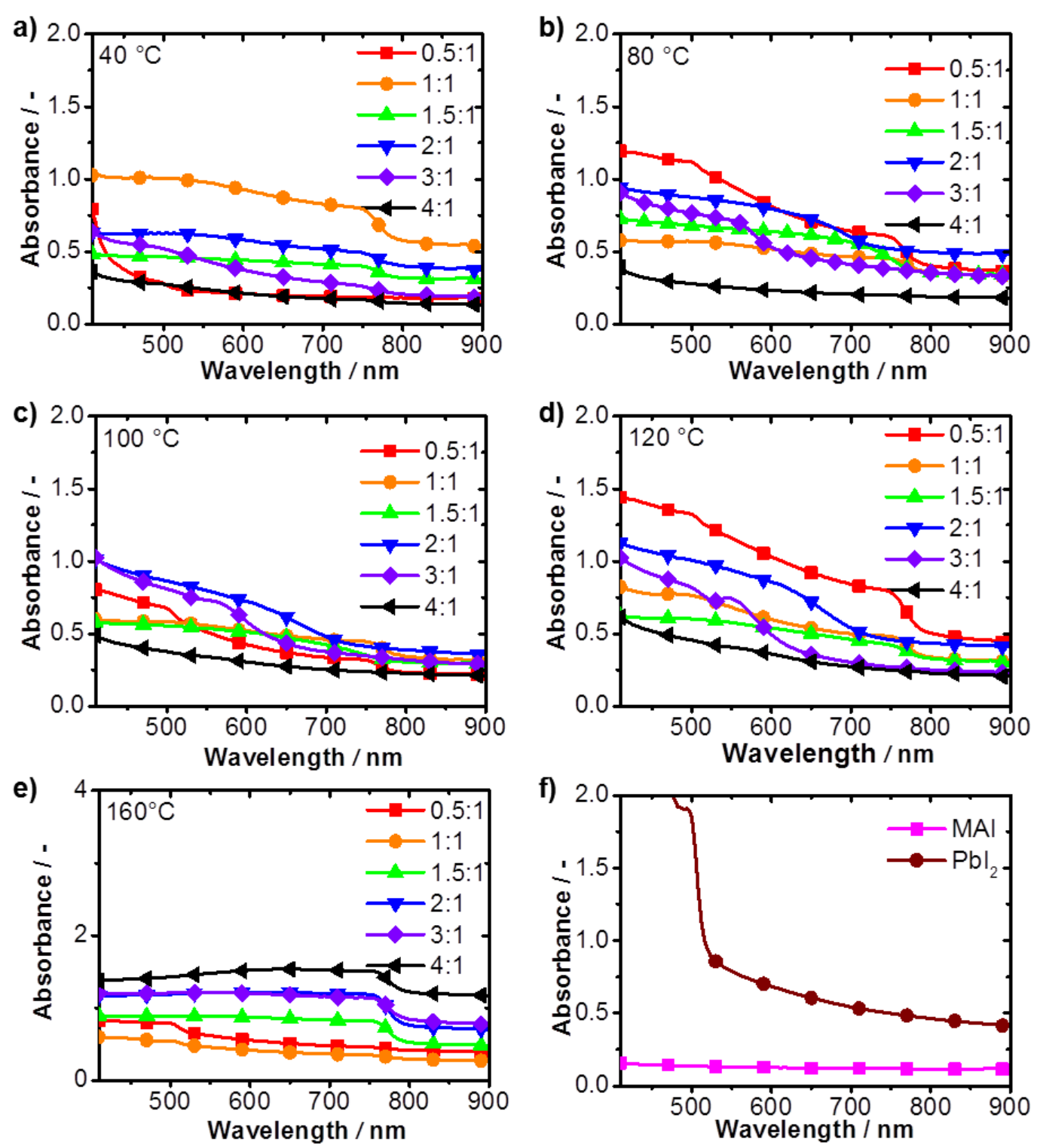

Figure S3: a)-e) Exemplary absorption spectra for different temperatures and molar ratios MAI: $\mathrm{PbI}_{2}$. f) Absorption spectra for pure educt materials after annealing at $100{ }^{\circ} \mathrm{C}$ for $1 \mathrm{~h}$. The concentrations of the deposited solutions are similar to the substances in the 1:1 ratio. 


\section{S4 Photoluminescence data}

In Figure S4 a)-g), the photoluminescence (PL) emission spectra are summarized for the different ratios and annealing temperatures. Again, for every condition one example out of a total of three measurements is plotted. The pure educt and substrate emission spectra are shown in Figure S4h together with a spectrum of a pure glass substrate without any coverage. 

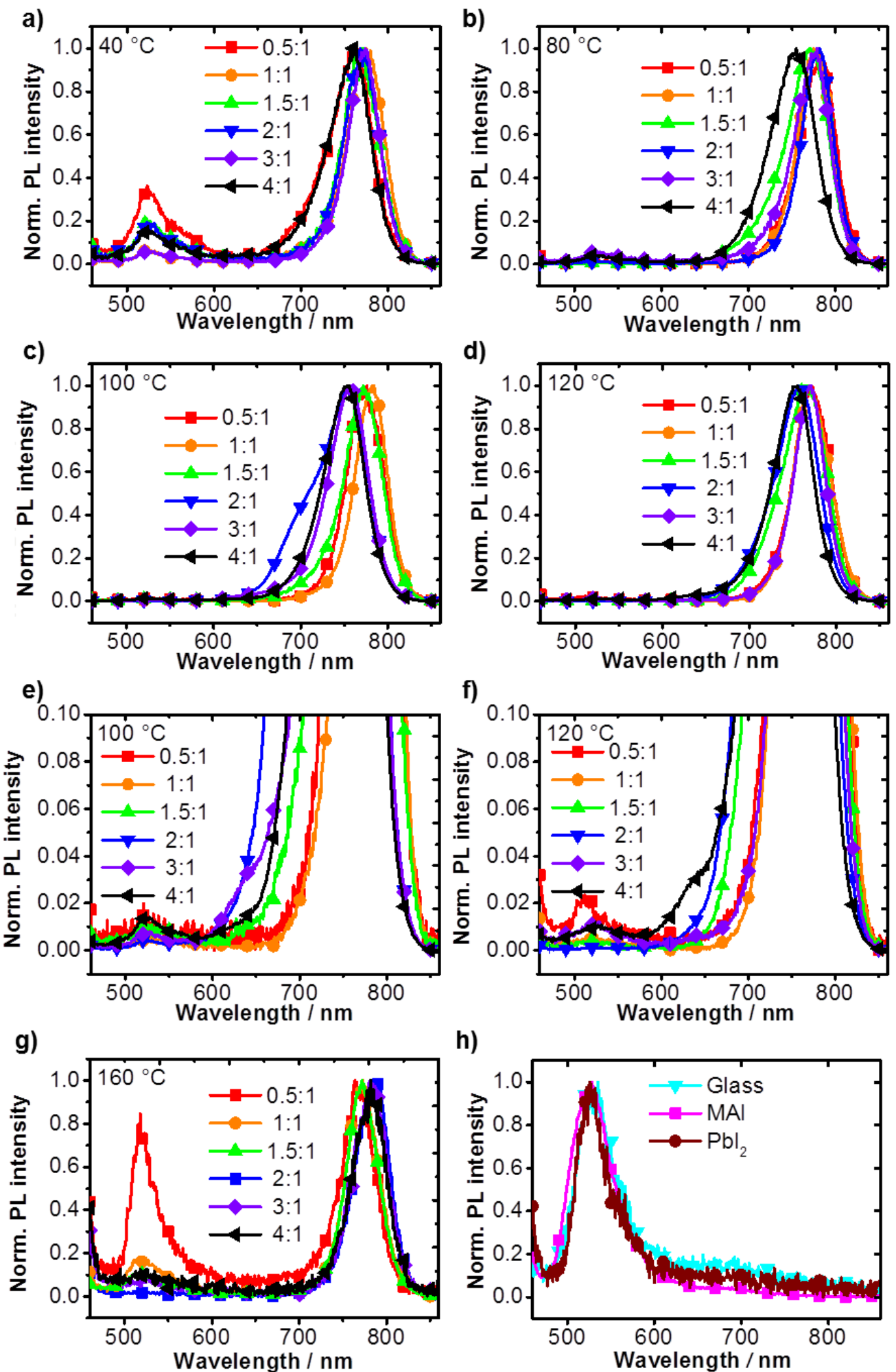

Figure S4: a)-d) and g) Exemplary normalized PL spectra for different temperatures and molar ratios of MAI:PbI ${ }_{2}$. e)-f) Magnification of the PL spectra at 100 and $120{ }^{\circ} \mathrm{C}$ to show the signal at $525 \mathrm{~nm}$. h) PL spectra for pure educt materials annealed at $100{ }^{\circ} \mathrm{C}$ for $1 \mathrm{~h}$. The used amounts of solid material of $\mathrm{PbI}_{2}$ and MAI were similar to the 1:1 ratio. 


\section{S5 X-ray diffraction data}

In Figure S5 a)-e) the X-ray diffraction (XRD) patterns are summarized for the tested reactant ratios and annealing temperatures. For better comparison, data for the pure educts is provided in Figure S5f.
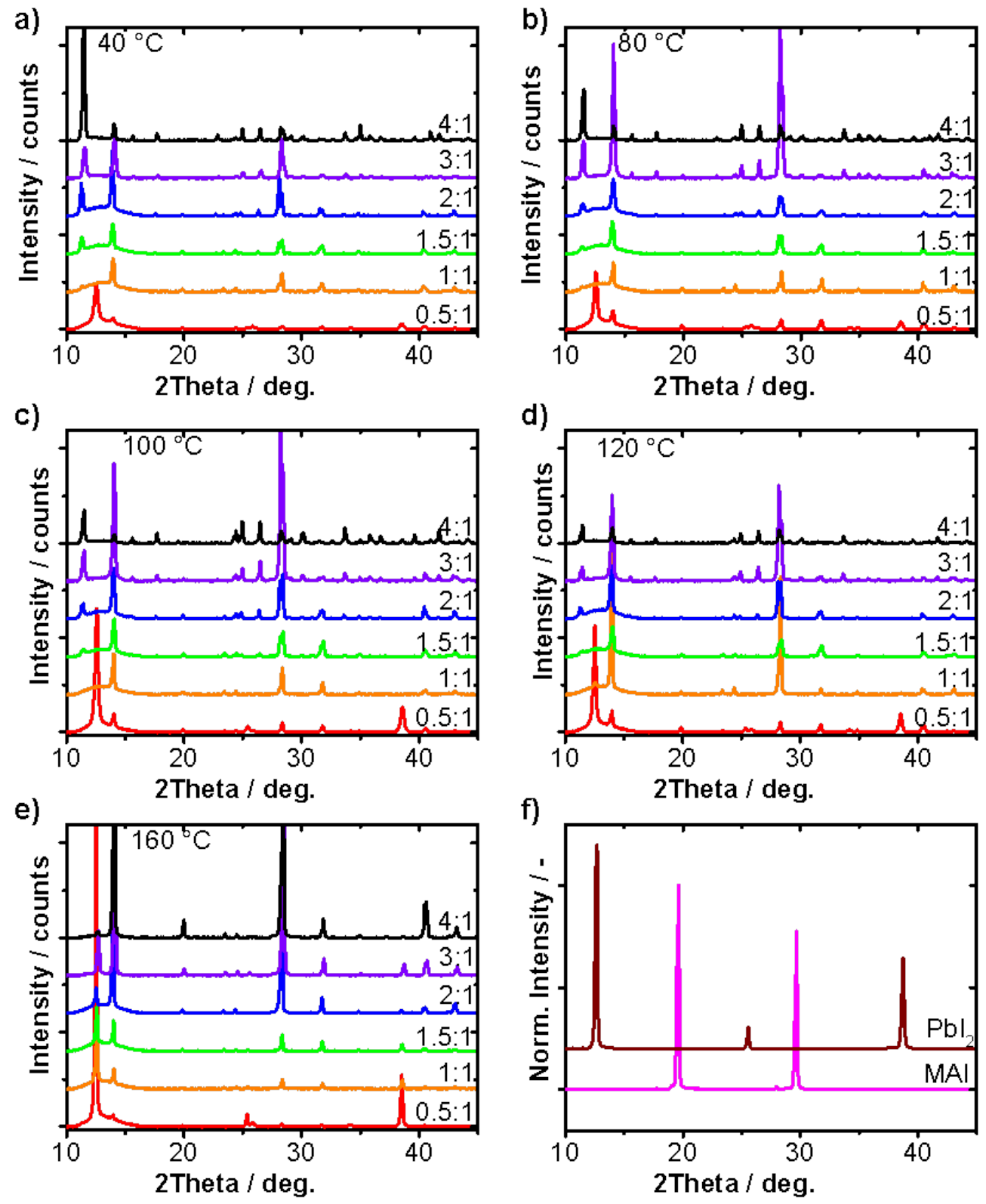

Figure S5: a)-e) XRD patterns for different annealing temperatures and molar ratios MAI:PbI ${ }_{2}$ f) XRD pattern for pure educt materials annealed at $100{ }^{\circ} \mathrm{C}$ for $1 \mathrm{~h}$. 


\section{S6 Fitting details of the model}

Model fitting can be summarized in four key statistical quantities. i) $\mathrm{R}^{2}$ (percent of variation), ii) $\mathrm{Q}^{2}$ (predictive ability), iii) model validity (measure of validity of the statistical model based on the input data), and iv) reproducibility (spread of the data under identical input conditions). To set things into perspective, in a perfect model, all values would be equal to 1. This means, perfectly linear behavior, experiments follow a very exact trend and identical outputs are obtained from the same parameters. However, in real-world experiments, the output varies due to the fluctuations in the experimental conditions. In case of our study, perovskites were drop-casted by the robot, however on glass slides with slightly different wetting behavior and then transferred to a hotplate, with comparatively less controlled evaporation. This is particularly holding true at the limits of the parameter space where very low or very high temperatures (e.g. 40 or $160{ }^{\circ} \mathrm{C}$ ) and ratios $(1: 0.5$ or 1:4) yield high deviations in the output. The values of $\mathrm{R}^{2}, \mathrm{Q}^{2}$, model validity and reproducibility are 0.6, $0.51,0.39$ and 0.64 respectively. To set things into perspective, for a good model, values

are of $\mathrm{R}^{2}$ and $\mathrm{Q}^{2} \geq 0.5$ and $\mathrm{R}^{2}-Q^{2} \leq 0.3$, model validity $\geq 0.25$ and reproducibility $\geq 0.5 .^{2}$ Thus, the model developed herein is absolutely satisfactory. 


\section{S7 Reduction of experimental time}

Table S1 summarizes the values for the estimated time of the different characterization techniques. To this end, the time for the measurement itself was ignored as this could be done without the operator being present and only the time for data evaluation was considered. The data sets were calculated sample-based, i.e., the time needed for a measurement of a single sample. For example, for absorbance measurements 24 samples could be processed in one run. Therefore the time for sample preparation and evaluation was divided by 24 . By using only the PL results, the evaluation time could be reduced up to $60 \%$.

Table S1: Estimated times for the preparation and evaluation of one sample, which has to be afforded by the experimenter.

\begin{tabular}{lc|c|cc}
\hline & \multicolumn{3}{c}{ Time/min } & Overall \\
\cline { 2 - 4 } & $\begin{array}{c}\text { Measurement } \\
\text { preparation }\end{array}$ & $\begin{array}{c}\text { Evaluation of } \\
\text { sample }\end{array}$ & $\begin{array}{c}\text { Percentage } \\
\text { portion of time } \\
/ \%\end{array}$ \\
\hline Absorbance & 0.4 & 0.4 & 0.8 & 9 \\
PL & 3.0 & 0.5 & 3.5 & 40 \\
XRD & 1.9 & 2.5 & 4.4. & 51 \\
\hline
\end{tabular}

\section{References}

(1) Li, B.; Isikgor, F. H.; Coskun, H.; Ouyang, J. The Effect of Methylammonium Iodide on the Supersaturation and Interfacial Energy of the Crystallization of Methylammonium Lead Triiodide Single Crystals. Angew. Chem., Int. Ed. 2017, 56, 16073-16076.

(2) MODDE 11 User Guide. 2015. 